\title{
Chapter 17 \\ ESSAI D'ANALYSE DES PHENOMENES INTERVENANT DANS LA FORMATION D'UN ESTUAIRE
}

\author{
M. BanaI \\ Directeur-Adjoint des Etudes et Recherches \\ Electricité de France
}

\section{INTRODUCTION}

Les considérations qui vont etre développées résultent des observations, des 6́tudes, et des lectures que l'auteur a pu faire pendant qu'il était chargé du Service des accès au port de Rouen sur le fleuve Seine au nord-ouest de la France.

II en résulte que $d^{\mathfrak{t} u n e}$ part elles ne valent que pour les estuaires possédant avec celui de la Seine les caractéristiques conmunes qui seront définies ci-dessous, mais $d^{2}$ autre part $q u^{1}$ on $s^{2}$ est efforcé $d^{2}$ en exclure ce qui est apparu comme trop particulier au cas de la Seine.

Les conditions auxquelles doit satisfaire un estuaire pour $\mathrm{qu}^{2}$ on puisse lui appliquer les considérations qui suivent, sont :

- forte marée et fạible débit fluvial,

- embouchure embarrassée de bancs découvrants constitués par du sable fin ou très fin (granulométrie voisine de $1 / 10 \mathrm{de} \mathrm{mm}$ ).

\section{MBCANISME DE LA FORMATION DES BANCS EN DE L'EVOLUTION DES CHENAUX}

La topographie $d^{2}$ un estuaire peut être examinée sous les trois aspects suivants :

- niveau moyen général des fonds,

- formation et érolution des chenaux et des bancs,

- largeurs et profondeurs des chenaux utilisables par la navigation.

NIVEAU MOYEN GEENERAL DES FONDS.

Si I'on dispose de levés des fonds assez anciens, on constatera en général une remontée progressive des bancs traduisant une accumulation de sable dans $I^{2}$ estuaire.

Cet engraissement séculaire $n^{3}$ est pas un phénomène extraordinaire en soi, car le colmatage des baies paraft être au contraire une règle assez générale $d^{2}$ évolution des côtes, dès lors que les courants ou la'houle trouvent des matériaux à mettre en suspension.

Dans le cas des estuaires, ce colmatage ne peut cependant être complet en raison du débit fluvial si faible soit-il. 


\section{ESSAI D'ANALYSE DES PHENOMENES INTERVENANT DANS LA FORMATION D'UN ESTUAIRE}

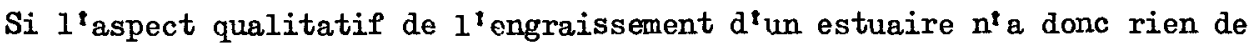
surprenant, on ne peut prévoir au contraire le taux annuel $d^{t}$ engraissement et la cote diétablissement des dépôts.

Lieu des dépôts - Les matériaux accumulés dans un estuaire sont répartis dans des dépôts de trois sortes (fig. 1) :

- zones ayant atteint la cote des P.M. de vives-eaux moyennes et couvertes en général $d^{\&}$ une écorce herbée,

- zones d'évolution des chenaux,

- zones de progression des bancs vers le large.

$\left.1^{\circ}\right)$ Bancs herbés : Une extension rapide des bancs herbés est en général la conséquence de constructions $d^{t}$ ouvrages protégeant certains bancs de l'action des courants. Toutefois, les bancs herbés peuvent progresser à partir de l'amont de l'estuaire et $s^{i} i l s$ ne sont pas protégés par des digues être attaqués par les courants et disparâtre après plusieurs années d'existence. Il faut cependant noter que la reprise des bancs dont le niveau atteint la cote de P.M. de V.E. qui ne peut se faire que par attaque du talus est toujours lente. Ces bancs sont donc relativement stables.

$\left.2^{\circ}\right)$ Zone d'évolution des chenaux : C'est dans cette zone que $s^{\prime}$ observent les fluctuations rapides des chenaux. Si le niveau moyen de cette zone d'érolution des chenaux $s^{t}$ élève progressivement sous $1^{t}$ effet de $1^{t}$ engraissement séculaire, on peut dire qutil est stable par rapport aux phénomènes se produisant pendant un petit nombre de marées.

Le flot provoque un déplacement de matériaux vers $1^{\text {tamont et le jusant }}$ effectue le transport contraire mais sans ramener les matériaux exactement là où ils ont été pris; le déplacement résiduel $\mathbf{s}^{\imath}$ efface sur une période plus longue par suite de la fluctuation des chenaux pour ne laisser comme bilan

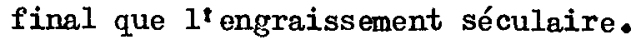

$\left.3^{\circ}\right)$ Zone de progression des bancs vers le large : Au large de la zone ci-dessus on observe des bancs (toujours inmergés) dont la topographie ne se déforme que lentement.

Ces bancs se déreloppent devant le débouché des chenaux de jusant et se rétractent ailleurs.

En moyenne, on observe une avancée progressive de ces bancs, conséquence de l'engraissement séculaire sauf pendant les périodes où des endiguements sont exécutés à 1 'amont.

Pendant ces périodes en effet, les bancs s'érodent de manière à fournir les matériaux qui s' accumulent derrière les digues.

Engraissement séculaire - Le taux annuel de l'engraissement de l'estuaire dépend grandement de la zone sur laquelle porte la comparaison.

Si l'on observe seulement la partie amont, l'engraissement est en général

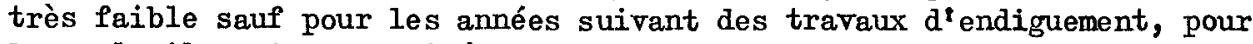
lesquels il devient considérable. 


\section{COASTAL ENGINEERING}

Si les cubctures couparatives portent sur tout l'ensemble de l'estuanre jusqu'au large (cote - 10 sous le niveau des BM par exenple), on constate au contraire un taux annuel $d^{z}$ engraissement assez peu variable $d^{\sharp}$ une année à l'autre, qu'on a tendance à considérer conme une constante de la nature.

Avant d'admettre toutefois $1^{1}$ existence $d^{\prime}$ un taux permanent $d^{t}$ engraisse-

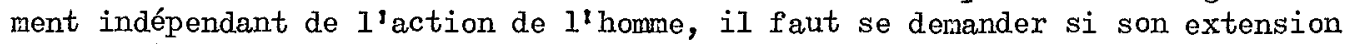
pendant 1000 ans ou davantage ne soulève pas des contradictions évidentes.

Usure des matériaux dans I'estuaıre - Il était presque unanimement admis autrefois que le sable narin était formé par un broyage progressif, par $1^{t}$ agitation de la mer, à l'état de plus en plus fin, des prodults de destruction de côtes.

On a plutôt tendance à considérer actuellenent que la granulonétrie des sables siliceux est celle des cristaux de silice du granit dont ils sont les produits de décomposition.

Par conséquent, aucun broyage des sables silıceux ne pourrait se produir dans $I^{\text {lestuaire et }}$ il $\mathrm{y}$ aurait une séparation complète entre le sable siliceux et la vase.

Il $n^{\prime} y$ aurait donc pas lieu de compter sur le broyage des ratériaux pour combattre $1^{\prime}$ engraissement $d^{t}$ un estuaire corme on $1^{t} a$ cru autrefois, et on ne devait attribuer aucun avantage à ce point de vue à l'existence d'une grande surface de bancs mobiles.

FORMATION ET EVOLUTION DES CHENAUX ET DES BANCS - DISSYMETRIE DES ACTIONS DU FLOT ET DU JUSANT.

Le courant de flot se forme alors que le niveau a déjà sensıblenert mont et atteint sa valeur maximum pour une cote assez voisine de la pleine-mer. Le jusant au contraire agit surtout au-dessous du niveau de la mı-marée (fig。2).

L'action du flot consiste donc dans un décapage général de tout l'estuair se produisant de manlère sensiblement égale sur les bancs et les chenaux, suivj $\mathrm{d}^{\mathfrak{I}}$ un dépôt uniforme à $1^{\mathbb{Q}}$ étale de pleine mer.

Le jusant au contraire n'agit sur les bancs qu'au début et son action se concentre rapidement dans les thalwegs.

Il. en résulte deux conséquences :

a) Les matériaux transportés par le jusant arrivent à la limite de I'estuaire par les chenaux et forment des barres $d^{\natural}$ embouchure aux débouchés de ces chenaux.

Au contraire, I'action de décapage du flot est unıforme sur la lısière aval des bancs.

Les fonds étant en moyenne en équilibre, il en résulte que des bancs se développent au débouché des chenaux et que des fosses se creusent ailleurs. 
ESSAI D'ANALYSE DES PHENOMENES INTERVENANT DANS LA FORMATION D'UN ESTUAIRE

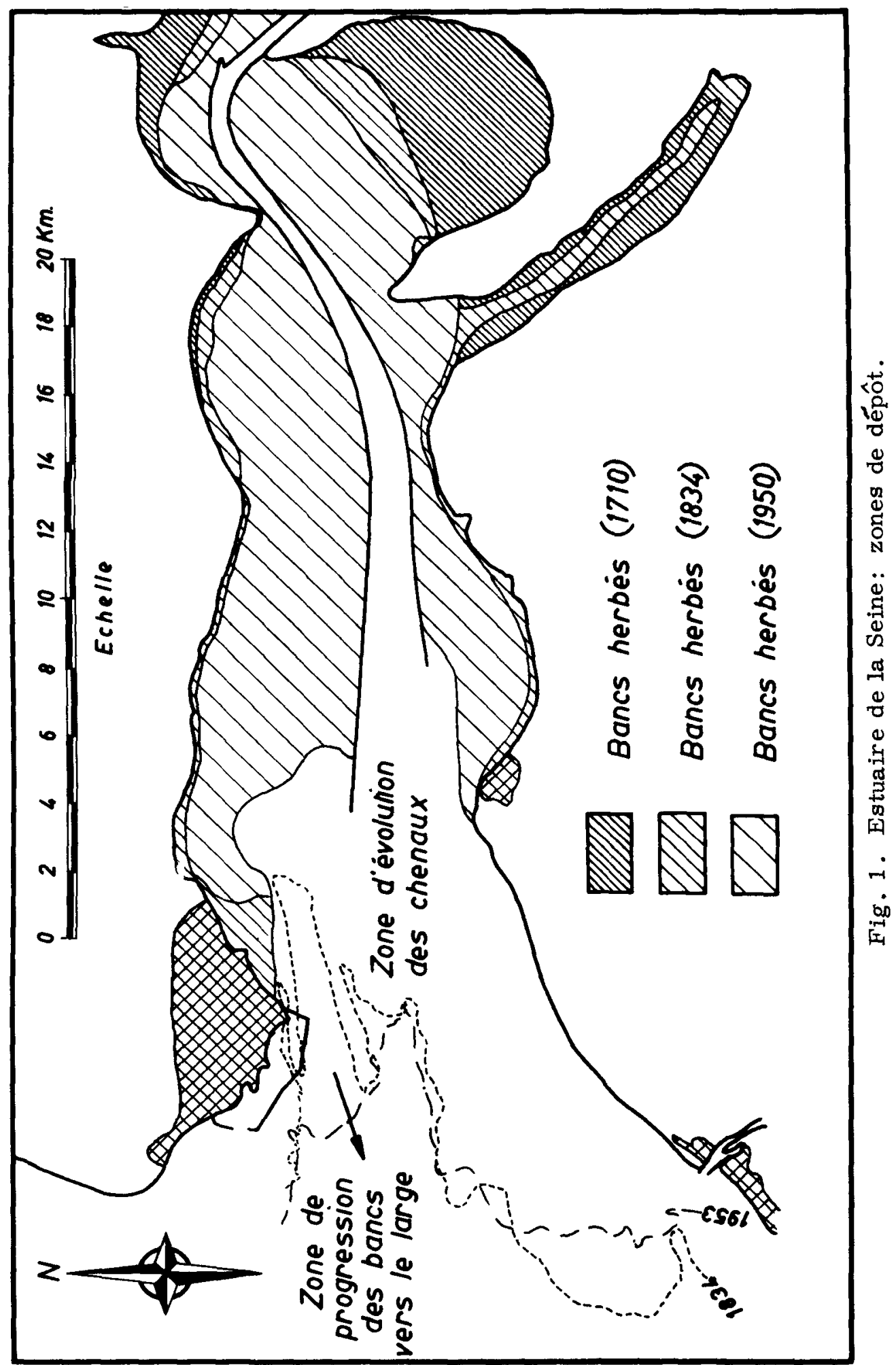




\section{COASTAL ENGINEERING}

$L^{2}$ équilibre de $I^{2}$ estuaire ne peut donc être obtenu que par les fluctuations des chenaux qui les font déboucher là où des fosses existaient précédemment.

Si cet effet $d^{2}$ auto-colmatage des chenaux est connu depuis très longtemI il ne semble pas que son importance ait toujours été complètement appréciée. Le cube des matériaux qui se déposent au débouché $d^{*} u n$ chenal à partir du moment où il $\mathrm{y}$ a une concentration totale du débit de fin de jusant peut être considérable et hors de proportion avec le taux annuel moyen $d^{\mathfrak{y} e n g r a i s s e m e n t}$ avec lequel il n'a a priori aucune relation.

Nous pensons que certaines fermetures brutales de chenaux imputées autrefois aux conditions atmosphériques n'étalent que le résultat du phénomène $d^{2}$ auto-colmatage.

b) Sauf à la lisière aval des bancs, le modelé des bancs et des chenaux résulte surtout de l'action du jusant.

Les érolutions des chenaux sont donc assez analogues à celles observées

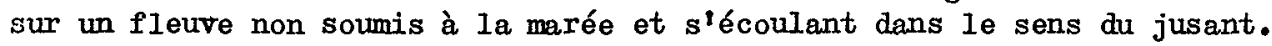

On sait depuis Fargue que la disposition du lit $d^{t} u n$ fleuve $s^{1}$ écoulant dans un terrain affouillable indéfini en $1^{2}$ absence de tout ouvrage diendiguement est une suite de méandres.

Les évolutions d'un tel lit sont de deux sortes :

- Evolutions progressives (accroissement de I'amplitude des méandres ou déplecement vers I'aval),

- Evolutions brutales, formation des coupures (lorsque le débouché aval $d^{\imath} u n$ chenal est colmaté).

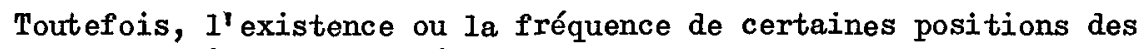
méandres peuvent etre influencées par les courants de flot dont la direction dépend notamment des courants au large et du tracé des berges de $I^{t}$ estuaire.

Il semble bien, par contre, que les tempêtes et les crues n'aient sur le modèlé des fonds de $I^{2} e s t u a i r e ~ q u^{\prime} u n e$ action réduite qu'il ne nous a pas été possible de constater d'une manière certaine.

\section{RESUME DES TENDANCES ESSENTIELLLES DE L' ESTUATRE.}

- Engraissement séculaire (formation des bancs herbés, engraissement du niveau général des bancs jusqu'à une certaine note moyenne, extension des bancs vers $I^{\text {'avall). }}$

- Chenaux existant à un instant donné se déformant à la manière du lit $d^{\prime} u n$ fleuve $s^{\prime}$ ćcoulant dans le sens du jusant.

- Remblaiement du débouché aval de tout chenal de jusant, le cube de matériaux déposés dépendant du décapage des bancs par le flot et non du taux $d^{2}$ engraissement séculaire de $I^{i}$ ensemble de $I^{t}$ estuaire. 


\section{ESSAI D'ANALYSE DES PHENOMENES INTERVENANT \\ DANS LA FORMATION D'UN ESTUAIRE}
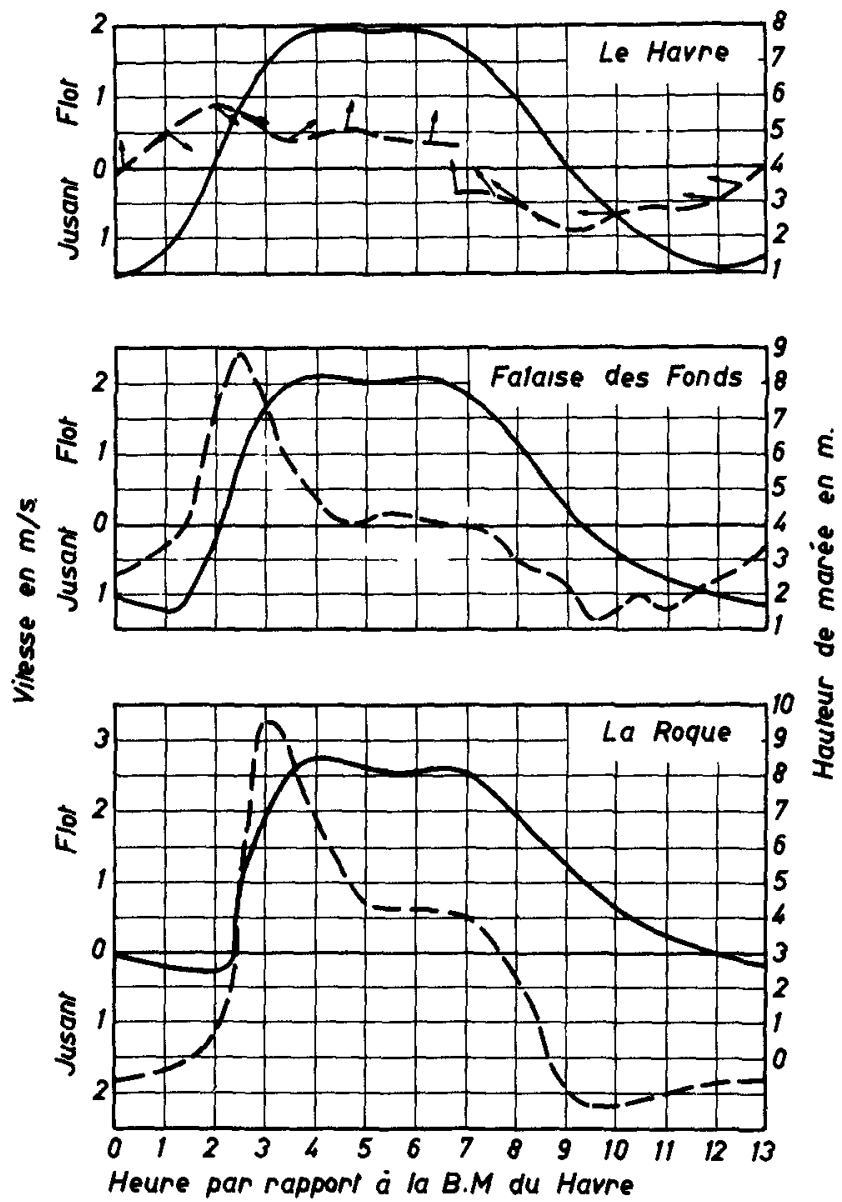

Fig. 2. Estuaire de la Seine: courbes marégraphiques et de vitesse de courant par coefficient 95 au Havre, à la Falaise des Fonds et à Ia Roque (Rive gauche).

(courbe de marée en trait plein___, courbe des vitesses en trait interrompu - - - - - ).

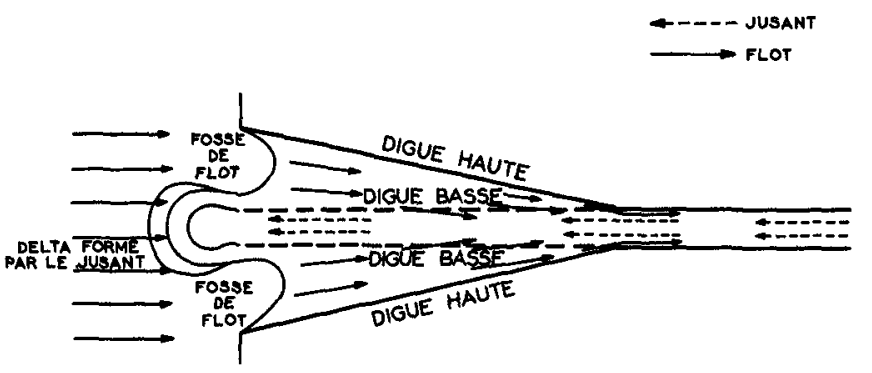

Fig. 3. Schéma des transports de matériaux dans un estuaire en entonnoir à endiguement de lit mineur. 


\section{COASTAL ENGINEERING}

- Echancrures des bancs à l'aval par le flot en dehors des débouchés des chenaux de jusant.

- Pérıodiquement, soit en raison du développeraent des méandres, soit en raison du colmatage à $1^{\prime}$ aval, formatı on $d^{l} u n$ nouveau chenal par ouverture brutale d'une coupure.

\section{HISTORIQUE DES EVOLUTIONS DE L'ESTUATRE}

Les règles élémentaires c1-dessus suffisent à peu près, dans une situatio donnée des ouvrages $d^{8}$ endiguement, à expliquer les évolutions à caractère sensiblement périodique constatées dans un estuaire sans qu'il soit nécessaire de faire intervenir les phénomènes aléatoires corme les tempêtes, les crues et encore moins le pur hasard.

Lorsque ces ouvrages sont modifıés, les évolutions se produisent dans de: conditions différentes.

Mais on peut constater pendant et après la constructıon des nouveaux

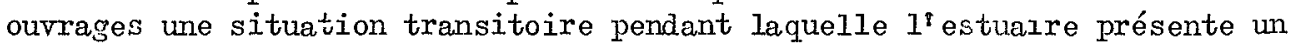
aspect "anormal".

Etant domée la fréquence des interventıons, un estuaıre peut rarement être considéré come évoluant librenent, et les évolutions des fonds constıtuant des séquelles des traveux ont souvent masqué les lols réglant les mouvements des chenaux indiqués précéderment.

Il faut précıser égalenent que les évolutions possıbles du chenal dépendent de l'état d'engraissement de $I^{\prime}$ estuaire et des évolùtıons antérıeures.

PROFONDEUR DES CHENAUX.

PRINCIPES DE L'AMELIORATTON DE L'ESTUATRE.

Nous étudierons simultanément les conditions qui fixent le profondeur

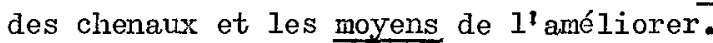

La profondeur d $\mathrm{d}^{\mathrm{t} u n}$ chenal utile à la navigation dépend de sa profondeur moyenne et de son modelé.

Un chenal de falble profondeur moyenne nais auquel une digue ì forte courbure donne un profıl en travers trıangulaire peut être plus favorable à la navigation qu'un chenal de profondeur noyenne supérıeure, maıs à fond plat.

De même des nouilles très profondes sont sans ıntérêt sı elles sont sćparées par des seuils d'infllexion plus élevés.

Les principes $\mathrm{d}^{\mathbf{t} a m e ́ n a g e n e n t ~ i n d ı q u e ́ s ~ p a r ~ F a r g u e ~ e t ~ G i r a r d o n ~ d o ı v e n t ~ d o n c ~}$ êtrre appliqués au tracé des endiguements. Mals une profondeur satisfalsante du chenal de navigation sera d'autant plus facilement obtenue que la profondeur moyenne du chenal hydraulique et sa largeur seront plus grandes.

Si, d'autre part, on $\mathrm{s}^{8}$ astreint à limiter le prolongenent des digues vers l'aval, on se trouvera tôt ou tard dans l'obligation d'utilıser un chenal 


\section{ESSAI D'ANALYSE DES PHENOMENES INTERVENANT DANS LA FORMATION D'UN ESTUAIRE}

libre pour lequel on ne pourra plus agir que sur les facteurs conditionnant la profondeur moyenne, à $l^{2}$ exclusion de tout effet $d^{d}$ ouvrage sur le courant de jusant.

Or, la profondeur moyenne est liée notanment à la "puissance hydraulique" du fleure. Cette notion, à laquelle une importance exceptionnelle est attribuée depuis de très nombreuses années, mérite d’être exanınée en détail.

SOLIDARITE ENTRE LES DIFFERENTES PARTIES D'UN FLEUVE A MAREE - LOI DE L'ETLARGISSEMENT KILOMETRIQUE.

Nous citerons des extraits du méroire célèbre de Mengin-Lecreulx sur la puissance hydraulique des fleures à marée. (1).

"Les courants qui sont par eux-mêmes des agents de déblaiement, qui creusent et entretiennent des chenaux de navigation sont en même temps les agents de transport des matières qui les encombrent. Ils portent donc en eux-mêmes le mal et le remède, et le résultat utile dépend $d^{i}$ un certain équilibre dont la détermination constitue la dufficulté du problème".

"D'autre part, en vertu du mouvement des eaux, toutes les parties d'un fleuve sont solidaires conme celles d'un organisme vivant, aucune d'elles ne peut être modifiée sans une répercussion plus ou moins forte sur toutes les autres, lors donc qu'on étudie une amélıoration locale, il est essentiel de rechercher quelle sera, pour le tempérament du fleuve, la conséquence des mesures projetées".

"En vertu du même principe, lorsqu' on veut araélıorer un fleuve, il importe de faire concorder entre elles, et autant que possible $d^{\mathfrak{t}}$ exécuter simultanément toutes les amélioratıons partielles, de manière que leurs effets se renforcent les uns les autres".

"Nous tiendrons pour acquis qu' on abordera les dufficultés de $1^{\prime}$ emboucuure dans des conditions d autant plus favorables que le débit de rarée sera plus considérable.(2) et que l'importance de ce débit doit etre proportionnée à celle des difficultés à valncre, soit qu'elles proviennent du débit solide $d^{\prime}$ amont, soit qu'elles proviennent du débit solide d'aval, c'est-ì-dire des ma'uè̀res venant de la mer, qui se présentent à $1^{\prime} \in$ entrée du fleuve, y pénètrent avec le flot, en ressortent avec le jusant et dans cette lutte entre l'introduction et $l^{\prime}$ expulsıon, donnent lieu à un état d'équilıbre plus ou moins favorable ̀̀ la navigation".

Mengin-Lecreulx se fixe donc comre but $1^{\prime}$ aménagement du fleuve dans la partie amont où il est endıgué, en vue d'arriver au débit maximum consıdéré, a prıori, comme la condition du succès pour l'aménagenent de l'aval.

(1) 1880

(2) Bien entendu, il ne faut prendre en considération que le débit concentré dans un chenal unique. 


\section{COASTAL ENGINEERING}

Il remarque que pour obtenir le débit maximum, il faut avoir les plus grandes largeurs possibles avec les profondeurs nécessaires à la fois à la transmission des marées et à la navigation et les plus faibles vitesses compatibles avec l'obtention des profondeurs.

Supposant connues, cette vitesse minimum et la profondeur à obtenir, Mengin-Lecreulx détermine une loi d élargissement de $1^{t}$ amont vers $1^{t}$ aval qui est de la forme $(1+\lambda)^{x} \quad(x$ abscisse en $\mathrm{km}, \lambda$ coefficient d'élargissement kilométrique).

Pour la vitesse de $0,8 \mathrm{~m} / \mathrm{s}$ admise par Mengin-Lecreulx, il trouve $\lambda=0,05$ environ, soit un élargissement de $50 \%$ tous les 10 kilomètres.

$L^{\prime} i d e ́ e ~ q u^{2} e n$ fonds affouillables indéfinis seul un estuaire ayant une certaine loi d'élargissement de l'amont vers l'aval conserve la loi de profondeur désirée nous paraît incontestable, mais la formule donnée par Mengin-Lecreulx reste à démontrer et la loi d'élargissement devract être fixée par des considérations expérimentales ou théoriques moins sonmaires. Cette notion doit en outre être complétée sur différents points que nous allons examiner.

\section{ENDIGUEMENT DU LIT MAJEUR.}

La loi d'élargissement kilométrique est établie par Mengin-Lecreulx en considérant seulement le jusant.

Il se borne à donner sur le flot les indications suiventes :

"De plus, puisque le niveau moyen pendant le flot est plus élevé que pendant le jusant, tout accroissement de largeur dans les parties supérieures diminuera la vitesse du flot sans affecter au même degré celle du jusant, il y aura donc bénéfice.

"Il est ainsi très utile à ce point de vue aussi bien qu'à celui de

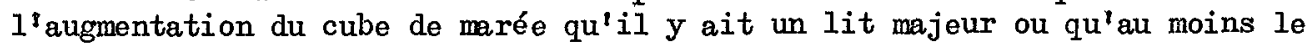
lit soit plus large à la surface qu'au fond. M. Franzius a fait remarquer $q^{\prime}$ 'au flot le niveau des eaux était convexe et que le lit central se déchargeait dans le lit majeur latéral tandis que pendant le jusant l'inverse se produisait, cette très intéressante observation conduit à la même conclusion".

Mengin-Lecreulx avait parfaitement compris que dans un estuaire comme celui de la Seine, le niveau d'équilibre des chenaux $s^{2}$ approfondissait si $l^{\prime}$ on réduisait la puissance de transport (et donc la vitesse) du flot par rapport à la puissance de transport du jusant.

Mais, il se trompe lorsqu'il pense pouvoir réduire le débit solide du flot en élargissant le lit majeur.

La vitesse instantanée du flot dans un profil en travers $n^{2}$ est pas conditionnée en effet par un débit déterminé à assurer, mais principalement par la loi locale de variation des hauteurs. 


\section{ESSAI D'ANALYSE DES PHENOMENES INTERVENANT DANS LA FORMATION D'UN ESTUAIRE}

En élargissant le lit au-dessus du niveau de basse-mer, on accrôtt donc le débit instantané du flot sans réduire les vitesses et par conséquent on accrôtt le débit solide.

Cependant, si les digues du lit majeur sont peu convergentes et sensiblement parallèles aux digues du lit mineur, $1^{\prime}$ observation de Franzius permet de penser que l'élargissement du lit majeur peut avoir un effet favorable sur la profondeur d'équilibre du chenal.

Mais, si les digues du lit majeur sont très convergentes, un résultat tout à fait contraire est obtenu.

Au lieu de la réduction de la vitesse du 1 lot escomptée par MenginLecreulx, on constate un accroissement très rapide de ces vitesses vers 1'amont.

Le cube accumulé à $1^{t}$ amont $n^{p}$ est que très falblement accru par la surélévation de la cote de P.M. au fond du convergent, mais la durée du

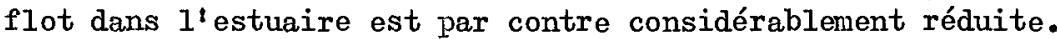

Les graphiques des vitesses montrent $1^{\prime}$ extrême brièveté de la pointe de vitesse du flot et la dissymétrie existant dans $1^{\prime}$ intérıeur de $1^{\text {l'estuaire }}$ entre le flot et le jusant.

Or, cette dissymétrie est directement opposée au but cherché puisqu'un cube liquide donné peut entraıner une charge solide d'autant plus forte qu'il est débité pendant un temps plus court.

Dans plusieurs études anglaises, la dissymétrie du jusant et du flot est considérée conme un véritable critère $d^{8}$ aménagement $d^{7}$ un fleuve à marée

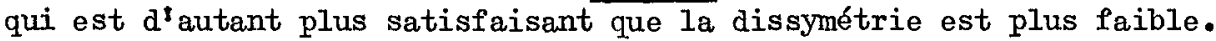

Par ailleurs, bien loin de se décharger dans le lit majeur latéral, le lit central reçoit les produits de décapage des bancs de lit majeur comme il apparaft clairement sur la figure 3.

$L^{1}$ idée de compléter un endiguement du lit mineur par des digues hautes beaucoup plus largement évasées, nous parâtt donc très fâcheuse.

\section{OBSERVATIONS COMPLEMENTATRES.}

a) Pour une loi donnée de marée, la dissymétrie entre le flot et le jusant est d'autant plus accusée que les profondeurs sont plus faibles. Autrement dit, un estuaire tend à se dégrader d'autant plus rapidement $q u^{t} i l$ part $\mathrm{d}^{x}$ une situation naturelle plus mauvaise.

b) La dissymétrie naturelle de la marée au large est une condition défavorabłe qui est probablement une des causes du niveau naturellement élevé des bancs de certains estuaires.

c) Même $s^{i} i l s$ ne sont pas limités par des digues très convergentes, les bancs du lit majeur peuvent constituer des dangers pour le chenal s'il n'est pas rectiligne. 


\section{COASTAL ENGINEERING}

Il n'est pas possible en effet de faire colncider la direction du courant de flot avec le tracé des digues basses de lit mineur.

Le déversement dans le chenal des matériaux arrachés aux bancs par le flot sera une cause de perturbation dans le chenal.

Il semble donc bien que le maıntıen d'un très large lit majeur présente de graves inconvénients; il ne $s^{\mathbf{l} a c c o m m o d e ~ n o t a m m e n t ~ p a s ~ d e s ~ i n f l e x i o n s . ~}$ Franzius était partisan de laisser subsister de larges lits majeurs et de faire des endiguements rectilimges, la coincidence de ces deux idées n'est peut-être pas fortuite.

\section{APPLICATION DE LA LOI D'ELLARGISSEMENT DE MENGIN-LECREUTXX - SIMTLITUDE.}

L'application pratique de la notion d'élargissement progressif vers 1 'aval parâ̂t devoır être limitée en général à une mise en harmonie des différentes parties d'un fleuve comportant seulement $I^{\prime}$ exécution de retouches (élargissement ou rétrécissement de certaınes parties d'endıguement, en consıdérant les autres comme acquises, dragages de fonds pas ou peu affouillables $(1)$, etc...).

Compte tenu des incertitudes dans la détermination théorique de la loi d'élargissement, on la choisıra en général d'après l'observation de la situam iion naturclle des fonds.

Le résultat obtenu sera certainement favorable aussı bıen dans la partie endiguée qu'à son aval.

Cependant, bien des ingénieurs ayant étudié l'anénagenent d'un estuaire ont falt observer qu'au lieu, de l'étrangler à l'aval, corme on le fait souvent Il serait bıon próférable de lui laisser son débouché naturel et de mettre l'enaiguement anont en harmonie avec ce débouché.

Il parât bıen probable en effet que, toutes choses égales d'ailleurs, des estuaires géométriquement semblables en plan (2) présentent des profondeurs d'autant plus grandes qu'ils sont plus Iarges. Toutefols l'incertitude actuelle sur le résultat quantitatif $d^{8}$ une dilatation transversale $d^{t} u n$ estuaire sur toute sa longueur a empêché jusqu'ic1 $d^{\prime}$ entreprendre les travaux considérables qu'elle nécessite (3)。

(1) L'endiguement doıt être conçu de manıère que les fonds s'entretiennent naturellement et non pour obtenir I'enlèvement naturel de dépốts anciens qui peuvent être beaucoup moins mobiles que les dépôts se formant à chaque rárée, bien que constıtués par le mêne matérıau.

(2) Pour pousser ce raisomnement par des considérations de similitude, on se heurte à deux difficultés du falt que le rarnage et le débit fluvial ne sont ias modifiés.

(3) Cet élargissement doit être prolongé vers I'amont jusqu'à la limite de pénétration des sables de mer existant après la transformatıon (qui peut être très en amont de la position de cette linıte avant la transformation). 


\section{ESSAI D'ANALYSE DES PHENOMENES INTERVENANT DANS LA FORMATION D'UN ESTUAIRE}

\section{REDUCTION DE L'AUTO-COLMATAGE.}

La concentration des apports de jusant au débouché des chenaux constitue une difficulté supplémentaire à vaincre pour établir des profondeurs satisialsantes au passage de la barre d'embouchure.

Il est entièrement conforme aux idées de Mengin-Lecreulx exposées précédemment que de penser qu' une réduction des apports solides du jusant provoquera un abaissement du niveau moyen du chenal.

Une réduction du débit de jusant dans le chenal pourra même être adriissible si elle est la condition $d^{\mathfrak{i}}$ une réduction relativement plus importante du débıt solide.

Enfin, $1^{\prime}$ efficacıté des dragages sera d'autant plus grande que les apports solides du jusant seront plus falbles.

Les considérations qui précèdent, conduısent à ajouter aux principes d'aménagement déjà exposés la réduction des apports du jusant au débouché du chenal par l'un des artıfices suivants :

a) Déviaition $d^{t}$ une partie du jusant en dehors du chenal de navigatıon,

b) Si $I^{\prime}$ anénagement comporte un chenal unique de jusant, utılisation de l'effet de courbure d'une digue de soutien du chenal d'où résulte une répartition des dépôts sur un banc de convexité où ils sont repris par le flot. flot.

c) Approfondissement local de la barre par un ouvrage agissant sur le

d) Réduction de la turbidité du jusant en supprimant le balayage des bancs par le flot.

\section{CONSEQUENCES DU PRINCIPE DtACCROISSEMENT DE LA PUISSANCE HYDRAULIQUE SUR LA CONCEPTION DES TRAVAUX D'AMENAGEMENT 。}

Nous solmes convaincus que tout ce qui sera fait pour mettre en hamone les unes avec les autres les différentes parties du chenal endigué aura certcinement un effet très favorable sur les conditions de navigation dans ce chenal endigué et sur la profondeur du chenal lıbre à l'aval des digues.

Nous avons Indiqué aussi qu' on pouvait escompter un résultat favorable de l'élargissement d'un estuaire sur toute sa longueur.

Nous ne pensons pas par contre que la considération de la puissance hydraulique puisse imposer une forme ou une autre pour $l^{\prime}$ endiguement de I'estualre.

Si les tracés dırects nous paraissent a priorı préférables aux tracés sinueux, c'est parce qu'ils évıtent des inflexions, mais mieux vaut certainenent un tracé sinueux stable qu'un tracé direct de $1^{\prime}$ endıguement du lit najeur que le chenal ne suit pas. 


\section{COASTAL ENGINEERING}

Par contre, une sinuosité supplémentaire utile à la stabilité ne saurai. réduire fâcheusement la puissance hydraulique.

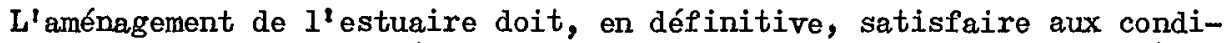
tions indiquées précédemment (stabilité et réduction de $1^{\prime}$ auto-colmatage) et si le souci d'accroftre la puissance hydraulique doit faire poursuivre activement les rectifications locales des irrégularités de $1^{\mathfrak{z}}$ endiguement à $1^{\prime}$ amont, il ne doit pas faire abandonner un tracé d'endiguement donnant, par ailleurs, les meilleures conditions au débouché aval du chenal.

Nous arons indiqué, $d^{2}$ autre part, que tous les moyens ne sont pas bons pour accrô̂tre la puissance hydraulique et que les bancs de lit majeur notamment accroissaient relativement plus le cube des matériaux apportés par le flot que le débit du jusant susceptible de les remporter. 